\title{
Machine learning methods can replace 3D profile method in classification of amyloidogenic hexapeptides
}

Jerzy Stanislawski ${ }^{1}$, Malgorzata Kotulska ${ }^{2^{*+}}$ and Olgierd Unold ${ }^{1 *+}$

\begin{abstract}
Background: Amyloids are proteins capable of forming fibrils. Many of them underlie serious diseases, like Alzheimer disease. The number of amyloid-associated diseases is constantly increasing. Recent studies indicate that amyloidogenic properties can be associated with short segments of aminoacids, which transform the structure when exposed. A few hundreds of such peptides have been experimentally found. Experimental testing of all possible aminoacid combinations is currently not feasible. Instead, they can be predicted by computational methods. 3D profile is a physicochemical-based method that has generated the most numerous dataset ZipperDB. However, it is computationally very demanding. Here, we show that dataset generation can be accelerated. Two methods to increase the classification efficiency of amyloidogenic candidates are presented and tested: simplified 3D profile generation and machine learning methods.

Results: We generated a new dataset of hexapeptides, using more economical 3D profile algorithm, which showed very good classification overlap with ZipperDB (93.5\%). The new part of our dataset contains 1779 segments, with 204 classified as amyloidogenic. The dataset of 6-residue sequences with their binary classification, based on the energy of the segment, was applied for training machine learning methods. A separate set of sequences from ZipperDB was used as a test set. The most effective methods were Alternating Decision Tree and Multilayer Perceptron. Both methods obtained area under ROC curve of 0.96 , accuracy $91 \%$, true positive rate ca. $78 \%$, and true negative rate $95 \%$. A few other machine learning methods also achieved a good performance. The computational time was reduced from 18-20 CPU-hours (full 3D profile) to 0.5 CPU-hours (simplified 3D profile) to seconds (machine learning).

Conclusions: We showed that the simplified profile generation method does not introduce an error with regard to the original method, while increasing the computational efficiency. Our new dataset proved representative enough to use simple statistical methods for testing the amylogenicity based only on six letter sequences. Statistical machine learning methods such as Alternating Decision Tree and Multilayer Perceptron can replace the energy based classifier, with advantage of very significantly reduced computational time and simplicity to perform the analysis. Additionally, a decision tree provides a set of very easily interpretable rules.
\end{abstract}

Keywords: Amyloid, 3D profile, WEKA, Alternating decision tree, Neural network

\footnotetext{
*Correspondence: malgorzata.kotulska@pwr.wroc.pl;

olgierd.unold@ pwr.wroc.pl

${ }^{\dagger}$ Equal contributors

${ }^{2}$ Institute of Biomedical Engineering and Instrumentation, Wroclaw University

of Technology, 50-370 Wroclaw, Poland

${ }^{1}$ Institute of Computer Engineering, Control and Robotics, Wroclaw

University of Technology, 50-370 Wroclaw, Poland

Full list of author information is available at the end of the article
} 


\section{Background}

Amyloids are proteins that can form fibrils - highly ordered aggregates of a characteristic zipper structure [1-4]. Majority of these proteins natively have a completely different functional structure in their physiological state, although functional amyloids also exist [5,6]. A hypothesis holds that in vivo amyloidogenic regions are usually capped by gatekeeper aminoacids, like prolines and glycines, which prevent aggregation, and may have a high affinity to chaperone proteins [7]. Very often amyloids lead to serious diseases, like Alzheimer disease (amyloid- $\beta$, tau), Parkinson disease ( $\alpha$-synuclein), type 2 diabetes (amylin), Creutzfeldt-Jakob disease (prion protein), Huntington disease (huntington), amyotrophic lateral sclerosis (SOD1), etc. (for a review see e.g. [5]). The number of diseases that turn out amyloid-associated is constantly increasing. It is believed that their toxicity is related to insertion of non-mature aggregates into plasma membranes as non-selective ion channels.

Recently, it was discovered that amyloidogenic properties can be due to short segments of aminoacids in a protein sequence (hot spots), which can transform the structure when non-burried [8]. It was proposed that hexapeptides can sufficiently represent such hot-spots, although they may vary between 4-10 aminoacids. A few hundreds of such peptides have been experimentally found, however testing all combinations is not possible. Instead, they can be predicted by computational methods.

Several physico-chemical methods have been proposed to predict amylogenicity of a peptide, e.g. Tango [9], ZipperDB [10,11], Pasta [12], AggreScan [13], PreAmyl [14], Zyggregator [15], CamFold [16], NetCSSP [17], FoldAmyloid [18], AmyloidMutant [19,20], BetaScan [21], and consensus AmylPred [22]. The majority of these methods predict probability of a sequence to form $\beta$-aggregates. As it turned out, such an approach was not always successful. Although $\beta$-aggregation is related to amyloidosis, structural and biophysical properties are different $[7,9]$. $\beta$-aggregation is quite common in highly concentrated proteins, which do not form fibers. On the other hand, certain amyloids, like prions, are poorly predicted by tools dedicated to $\beta$-aggregates.

Methods like 3D profile, applied in ZipperDB or AmyloidMutant, which take into account more specific structural features of amyloids - resembling a steric zipper [4] - work better in such cases. Also statistical elements seem to help in the classification, as shown in Waltz [23] using Position Specific Scoring Matrices (PSSM), or Bayesian classifier and weighted decision tree applied to long sequences of bacterial antibodies [24].

Experimental datasets, upon which new classification methods could be built, are still very limited. Those sequences that show amyloid propensity are rarely well characterized. For the majority of them, it is not known which segment is responsible for their amylogenicity and few of them have an experimental structure of high resolution [4]. The biggest database of potential hexapeptides, generated with the 3D profile method, comes from the ZipperDB. The classical 3D profile method applies over 2.5 thousand scaffolds resembling a steric zipper structure, on which tested hexapeptides are threaded, and their minimal energy is calculated. If the minimal energy of one chain is below a threshold value, which could be obtained from experimental dataset of hexapeptides, then the hexapeptide is classified as amyloidogenic. The method is reasonable and quite accurate - the authors of Waltz tested it on the independent dataset from prion protein sup35, which was experimentally derived. They reported that the 3D profile method showed accuracy of 0.8 , with sensitivity of 0.67 and specificity of 0.84 [23]. The database in ZipperDB, which is freely available on-line [25], is constantly growing. Currently it covers all ORFs from 3 genomes: H. sapiens, S. cervisiae, and $E$. coli, with $50 \%$ redundancy. Interestingly, the database shows hot spots in a majority of proteins. It does not mean that they can easily turn into amyloids in the physiological conditions but it shows new interesting aspects of this topic. Unfortunately, the 3D profile method is very computationally expensive and not very simple to use.

In this paper, we propose two methods to extend the ZipperDB dataset, classifying hexapeptide candidates at lower computational cost. One of the methods is closely related to the original idea of ZipperDB, only reducing the number of profiles. The other one, which introduces the main increase of the efficiency, uses a completely different statistical approach - machine learning. Both methods are tested versus original ZipperDB database classification.

\section{Results and discussion \\ Dataset}

We generated a new dataset of 4481 hexapeptdes, which was later used for training machine learning methods (see Additional file 1, trainset(+) and trainset(-)), using our version of 3D profile method with very significantly reduced number of profiles (see Methods - Dataset), and the method of exact energy calculation proposed in ZipperDB 2006 [10]. The dataset contains 825 positively and 3656 negatively classified segments. Part of our dataset (2702 hexapeptides, see Additional file 1) is also available in ZipperDB 2010 (as of February 2012), which uses the simplified "triplet" method of calculating the energy and fuzzy logic. Energies of these hexapeptides, obtained from our study and from ZipperDB, were compared. Based on the energy criterion, $93.5 \%$ of the segments were identically classified with regard to their amyloid properties (Additional file 1). In this set, 622 
(23\%) hexapeptides were classified by our energy value as amyloidogenic; ZipperDB classified 612 (22.6\%) as amyloidogenic. Differently classified hexapeptides often had energies close to the classification threshold of $-23 \mathrm{kcal} / \mathrm{mol}$. The mean absolute value of the energy difference, between these two sources, was $1 \mathrm{kcal} / \mathrm{mol}$; $90 \%$ of the difference was below $1.4 \mathrm{kcal} / \mathrm{mol}$. Energy values could differ because of limited number of threading profiles, as well as randomization element in Rosetta Design. Moreover, computationally faster but simplified triplet method, used in ZipperDB 2010, also affected the results. As stated by the ZipperDB authors, who tested it on segments of E. coli ORFs, an average error introduced by the triplet method was of $4 \mathrm{kcal} / \mathrm{mol}$ ( $90 \%$ of the difference) with the tendency to overestimate amylogenicity [11].

Our study shows that the results from our threading method are very comparable with those from ZipperDB 2010. In the new part of our dataset (1779 segments), 204 (11\%) of the hexapeptides were classified as amyloidogenic (Additional file 1). Also position dependant frequencies of each aminoacid are similar in our set and the set from ZipperDB (see Methods - Dataset and Additional file 2).

\section{Machine learning methods}

Our dataset, with a binary classification of sequence amyloid propensities based on their calculated energies, was applied for training machine learning methods [26], provided by WEKA [27]. Our objective was testing a potential for sequence based machine learning methods, which could be very significantly faster than threading and energy calculation. From a hundred of different machine learning methods, pre-selection was carried out (see Methods). Special consideration was given to methods with the highest potential for biochemical interpretation. From the preliminary tests, using cross-validation on the training set and selected efficiency measures, ten methods gave promising results: Alternating Decision Tree (ADTree) [28], Best-First Tree (BFTree) [29], Functional Tree (FT) [30], a clone of the Repeated Incremental Pruning to Produce Error Reduction (JRip) [31], a PART decision list (PART) [32], Ripple Down Rule (Ridor) [33], Support Vector Machine (SVM) method, implemented in WEKA with Sequential Minimal Optimization (SMO) algorithm for training a support vector classifier using polynomial or RBF kernels [34], MultiLayer Perceptron (MLP) [35], Naive Bayes [36], and Random Forest (RF) [37].

The final results of these 10 methods, using a separate test set (see Methods - Database) are shown in Table 1. The parameters of the methods (True Positive Rate - TPR, where amyloidogenic is regarded as "positive", True Negative Rate - TNR, Area Under ROC Curve - AUC) were optimized. Top methods were selected according to their AUC ROC (Figure 1).
Table 1 Machine learning performance

\begin{tabular}{ccccc}
\hline Method & TPR & TNR & Acc & AUC \\
\hline MLP & 0.78 & 0.95 & 0.91 & 0.96 \\
\hline ADTree 250 & 0.78 & 0.95 & 0.91 & 0.96 \\
\hline Naive Bayes & 0.53 & 0.98 & 0.88 & 0.95 \\
\hline ADTree 50 & 0.64 & 0.96 & 0.89 & 0.94 \\
\hline RF & 0.26 & 0.98 & 0.82 & 0.89 \\
\hline FT & 0.73 & 0.94 & 0.90 & 0.85 \\
\hline SVM & 0.76 & 0.95 & 0.91 & 0.86 \\
\hline Part & 0.56 & 0.94 & 0.86 & 0.85 \\
\hline BFTree & 0.67 & 0.91 & 0.86 & 0.82 \\
\hline Ridor & 0.56 & 0.90 & 0.83 & 0.73 \\
\hline Jrip & 0.29 & 0.93 & 0.79 & 0.61 \\
\hline
\end{tabular}

The performance evaluation of the machine learning methods. The results are ordered by decreasing AUC.

The tests showed that some of the standard WEKA methods can be very successfully used for classification of amyloidogenic segments, compatible with 3D profile method. In the best methods, Acc was typically close to 90\%. The most effective methods from WEKA were MLP, ADTree, and Naive Bayes (results in Table 1, model details in Additional file 3).

The best ADTree, with 250 rules, achieved AUC=0.96, which is close to maximum $\mathrm{AUC}=1$, characteristic of an ideal classifier, $\mathrm{TPR}=0.78, \mathrm{TNR}=0.95$, and $\mathrm{Acc}=0.91$. Identical results were achieved with MLP classifier. We have also tested if removal of hexapaptides that overlap experimental datasets and introduce a bias in sequences, coming from the highly redundant AmylHex dataset, influence the result of our classifiers. The ADTree 250 trained on the reduced training set, deprived of hexapeptides overlapping AmylHex and Waltz, showed higher efficiency - it obtained AUC $=0.98, \mathrm{TPR}=0.81$, TNR $=0.96$, and Acc $=0.94$. The rules (trained on the full set) concerning each hexapeptide position are presented and compared in Additional file 4. There are some differences between trees built on the full and reduced training sets. For example, the tree from the full training set favored valine at position 3 (rule 14 with factor 0.6, Additional file 4), overrepresented in AmylHex, while the tree built on the reduced dataset shows valine only in rule 47 (less significant), and with factor 0.45 . The trees with a lower number of rules, which are easier for interpretation, are also good classifiers (see ADTree with 50 rules in Figure 2). The top rules for this tree are fairly compatible with the PSSM underlying Waltz method reported in [23], and they indicate, for example, that isoleucine (I) is highly expected at position 4 (see ADTree with 50 rules in Figure 2, rule 1: AA4=I with the factor of 0.926 ), while proline (P) and arginine $(\mathrm{R})$ are not welcome (Figure 2, ADTree with 50 rules, rule 12: $\mathrm{AA} 4=\mathrm{P}$ with the factor of -2.077 , and rule 13:AA4=R with the factor of -1.636 , respectively). 


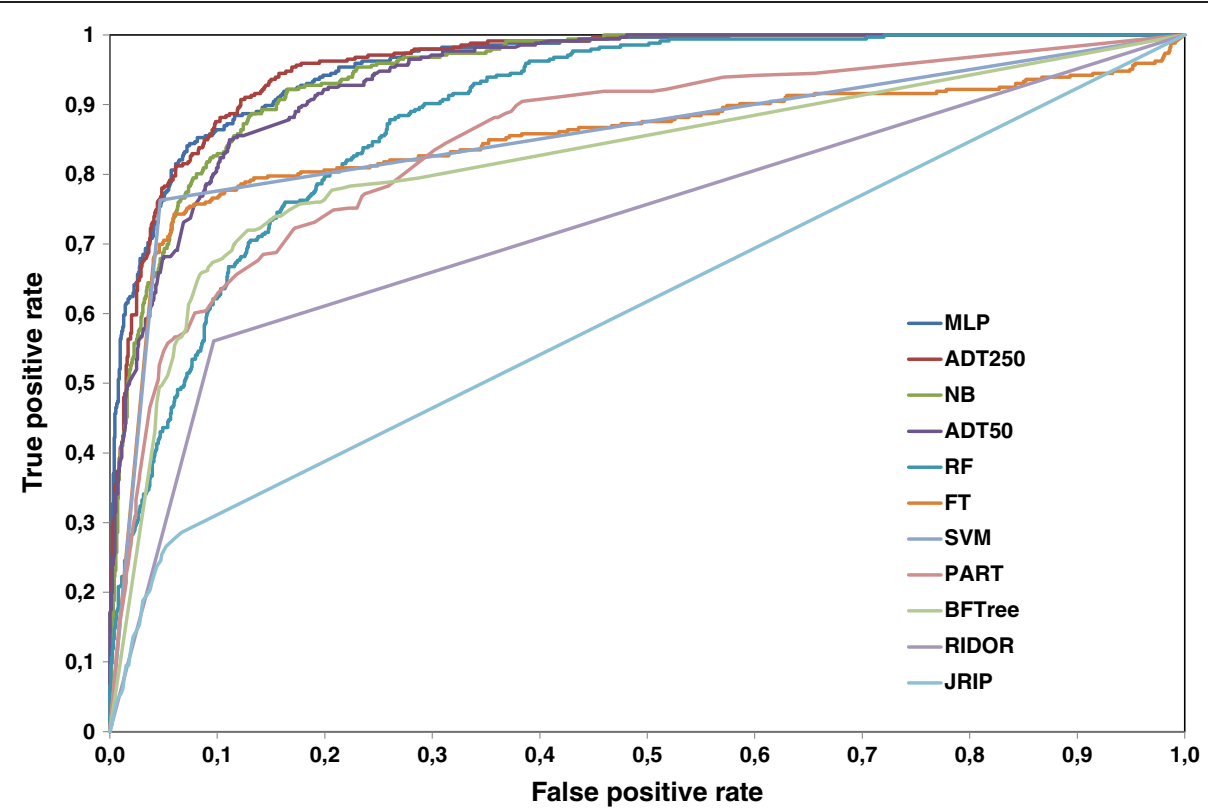

Figure $1 \mathrm{~A}$ plot of ROC curves of all methods. A plot of ROC curve for all the methods. Among all methods, MultiLayer Perceptron and Alternating Decision Tree with 250 boosting iterations cover the maximum area under the curve (i.e. 0.96), closely followed by Naive Bayes (AUC of 0.95) and Alternating Decision Tree with 50 boosting iterations (AUC of 0.94). In Table 1 all the corresponding AUC values are reported.

Efficiency of the MLP method was not sensitive to the presence of the redundant hexapeptides in the training set (results in Additional file 3). Naive Bayes improved its efficiency when trained on the reduced set $(\mathrm{AUC}=0.97$, $\mathrm{TPR}=0.54, \mathrm{TNR}=0.99$, Acc $=0.91$ ).
A representative estimate of WEKA methods performance, which was independent of class distribution and the specifics of the training data set (see Methods) is presented in Table 2, showing the number of wins, draws and losses when all methods are compared to each other

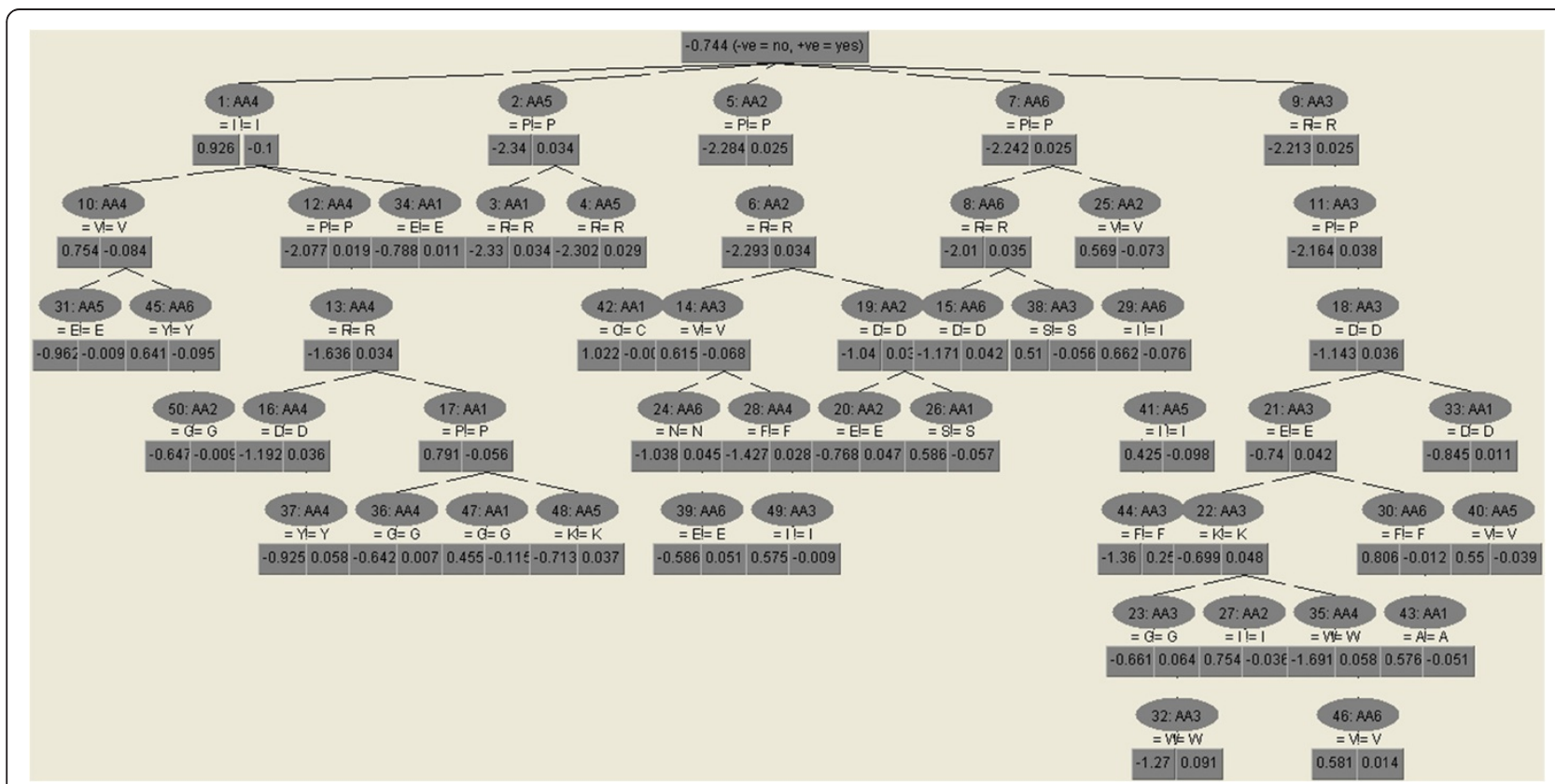

Figure 2 ADTree with $\mathbf{5 0}$ rules. In notation of the rules, $n$ : AA $j, n$ indicates the rule number (ordered by their significance), $j$ denotes the aminoacid position. Below the rule label, the aminoacid occurrence (or absence marked by "!") are valued by numbers. Negative numbers denote low aminoacid occurrence. A detailed explanation on how to read the Alternating Decision Tree is given in the main text. 
Table 2 Statistical evaluation

\begin{tabular}{cccc}
\hline Method & Wins & Draws & Losses \\
\hline MLP & 10 & 0 & 0 \\
\hline ADTree 250 & 8 & 1 & 1 \\
\hline Naive Bayes & 6 & 3 & 1 \\
\hline ADTree 50 & 6 & 2 & 2 \\
\hline FT & 3 & 5 & 2 \\
\hline SVM & 3 & 3 & 4 \\
\hline PART & 3 & 3 & 4 \\
\hline RF & 3 & 3 & 4 \\
\hline Ridor & 1 & 1 & 8 \\
\hline BFTree & 1 & 1 & 8 \\
\hline JRip & 0 & 0 & 10 \\
\hline
\end{tabular}

Summary of wins, draws and losses of each method confronted with others (with regard to their AUCs).

for AUC. The corrected paired t-test showed that ADTree and MLP had statistically significantly higher AUCs than other methods, at the $5 \%$ of the significance level.

\section{Compatibility of the energy based classification with FoldAmyloid and Waltz}

To test to what extent the 3D profile method overlaps with other state of the art methods, i.e. how universal its extended datasets could be, we performed the amylogenicity prediction with other tools: FoldAmyloid and Waltz, using different classification methods. FoldAmyloid is based on the packing density [23] and Waltz is based on PSSMs primarily derived from a dataset classified by physicochemical modeling [9]. The whole set of 4481 hexapeptides was tested, using all FoldAmyloid options and Waltz optimizations for overall performance and sensitivity (Additional file 5). The results are summarized in Table 3.

The overall classification overlap was similar in all methods; typically $80 \%$ of the hexapeptides were classified identically, using 3D profile and FoldAmyloid or Waltz. On the other hand, $84-88 \%$ of hexapeptides, classified by
3D profile as non-amyloidogenic, were also negatively classified by FoldAmyloid; Waltz overlap of negatives was 91-97\%. However, classification of the positive hexapeptides was less compatible - ranging from 11\% in Waltz best performance method to $43 \%$ in FoldAmyloid triple hybrid method. This result shows that classification of positive instances is more challenging and should become the target. Less numerous positive datasets of experimental data, on which all classification methods were previously trained, could contribute to this situation. Also in our dataset, only $18.4 \%$ of hexapeptides were regarded as positive. Importantly, recognition of non-amyloid segments in the optimal method overlapped in $84 \%$. This means that negative peptides can be eliminated efficiently and consistently between different methods. We have also tested the consensus between 3D profile, FoldAmyloid triple hybrid and Waltz high sensitivity. It turned out that the overlap was $69 \%$, in which positive rate was $21 \%$ and negative $65 \%$.

\section{Conclusions}

Extending of the hexapeptide dataset, with computationally effective methods, could help in predicting amyloidogenic regions without laboratory experiments, which are currently not possible on all sequence combinations. We proposed an optimization to the classical 3D profile method, and using only 54 arbitrarily selected profiles we generated the new dataset of hexapeptides classified with regard to their amylogenicity. Energies of our segments showed very good overlap with the segments currently available in ZipperDB, which used the simplified Triplet method to calculate the energy. The new part of our dataset contains 1779 segments that have not been previously considered, with 204 segments classified by energy value as amyloidogenic.

We also performed the amylogenicity prediction on our dataset, using different classification methods - FoldAmyloid and Waltz. The best result was obtained with FoldAmyloid triple hybrid method, which overlapped

Table 3 ZipperDB versus other methods

\begin{tabular}{cccc}
\hline Method & Overlap & Positive & Negative \\
\hline 1. FoldAmyloid Contacts & 0.77 & 0.36 & 0.86 \\
\hline 2. FoldAmyloid Bone-Bone Donors & 0.79 & 0.39 & 0.88 \\
\hline 3. FoldAmyloid Bone-Bone Acceptors & 0.78 & 0.39 & 0.86 \\
\hline 4. FoldAmyloid Contacts+Donors & 0.78 & 0.38 & 0.87 \\
\hline 5. FoldAmyloid Contacts+Donors+Acceptors & $\mathbf{0 . 7 7}$ & $\mathbf{0 . 4 3}$ & $\mathbf{0 . 8 4}$ \\
\hline 6. Waltz Best Performance & 0.81 & 0.11 & 0.97 \\
\hline 7. Waltz High Sensitivity & 0.80 & 0.30 & 0.91 \\
\hline CONSENSUS 3D profile $+(5)+(7)$ & 0.69 & 0.21 & 0.65 \\
\hline
\end{tabular}

Compatibility of the 3D profile classification with FoldAmyloid and Waltz. 
the 3D profile classification in 77\% (total), 43\% (amyloidogenic), and $84 \%$ (non-amyloidogenic). It showed that different methods are quite compatible in the elimination process, and in this respect datasets generated with the 3D profile methods are universal.

To test whether statistical approach, trained on our dataset, could replace the energy based classifier, we used machine learning methods implemented in WEKA. Our dataset of 6-residue sequences, with a binary classification of their amyloidogenic propensities based on the calculated energy, was applied for training. From all available WEKA methods, we selected those giving the best results and tested with a separate test set, obtained from ZipperDB. Our study showed that some of the methods could be very successfully used for classification of amyloidogenic segments, compatible to the 3D profile method. The most effective methods in WEKA, in terms of AUC ROC, were Alternating Decision Tree and a Neural Network of a Multilayer Perceptron architecture, both with AUC $=0.96$. The ADTree efficiency could be increased to AUC $=0.98$ when highly redundant set of experimental hexapeptides was removed from the training set. The performance was then very close to an ideal classifier, for which $\mathrm{AUC}=1$. A great advantage of ADTree method is a set of very easily interpretable rules. Part of the rules were fairly compatible with the PSSM underlying another classification method -Waltz, which was based on different data All those methods could classify almost $80 \%$ of positive and $95 \%$ of negative hexapeptides identically as the 3D profile method.

Such a good result of classification, based only on aminoacid sequence and its binary classification, is very interesting. It shows a good correlation between classification with the laborious 3D profile method using the minimal chain energy from numerous putative structures, and purely statistical machine learning methods - using just 6 letters and the binary classification. This is possible only if a strong statistical pattern exists in the amyloid sequences recognized by $3 \mathrm{D}$ profile. Our results also prove that our new dataset is representative enough for training machine learning methods, in order to obtain amylogenicity of new segments only based on their six letter sequences, with no need to carry out threading procedure and energy evaluation

The main advantage of the machine learning approach, presented in this paper, is very significantly reduced computational time. Instead of 18-20 CPU-hours with the full 3D profile method or $0.5 \mathrm{CPU}$-hours with the simplified 3D profile, the classification can take below 1 CPU-minute with a very good overlap of the results. Such a reduction of the computational time is crucial when large amount of hexapeptides should be classified. Additionally, the machine learning enhances the simplicity to perform the analysis.

\section{Methods}

\section{Database}

As a reference dataset of 6-residue sequences, also applied to test our machine learning results, we used the first set published in ZipperDB as of 2010 (Additional file 1: testset(+) and testset(-)) [11].

The set used for training machine learning methods was obtained from non-redundant protein sequences of UniProt [38], cut into 6-residue windows by shifting of 1 position along the full sequence. The hexapeptides were then divided into amylo-positive and amylo-negative candidates with our simplified 3D profile method. To increase chances of finding amyloid segments, UniProt entries containing the keyword "amyloid" and proteins from AmyPDB database $[39,40]$ were selected for the procedure (both accessed in September 2010). The sequences were first cut into strings not exceeding 80 aminoacids each, and excessive redundancy was reduced at the level of $90 \%$, with CD-HIT (Cluster Database at High Identity with Tolerance) [41,42]. Next, the remaining sequences were cut into hexapeptides with a window of length 6, shifted of 1 position in each move. The set was finally enlarged with 266 hexapeptides studied experimentally: AmylHex dataset [10] and Waltz [23].

The full dataset do not show position dependence of aminoacids, with statistics close to the frequencies of aminoacids in all UniProt [43]. Even closer statistics were obtained for the dataset not enriched with the hexapeptides from AmylHex and Waltz. The statistics of the test set is also close to the same characteristics, although some numbers slightly differ (e.g. prolines are excluded in the test set). Standard deviations are negligible in both datasets. The statistics of datasets, in the form of tables and logos (prepared with WebLogo [44]), are presented in Additional file 2.

\section{Threading and energy calculation}

First, all cysteines were replaced by serines to avoid disulfide bridges as in [10]. Similarly to 3D profile method [10], a fibril-forming peptide NNQQNY, from sup35 prion protein of Saccharomyces, was used as a scaffold in the threading method. Each hexapeptide from the set was threaded on the scaffold; 5 identical copies of the hexapeptide formed one of two identical $\beta$-sheets. In the final structure, one of the $\beta$-sheets was shifted relative to the other one. In our implementation, which differs from $3 \mathrm{D}$ profile method, the movement was exercised in two planes: along the chains of $0-8 \AA$ with the step of $1 \AA$, and across the sheets fixing the distance between them to $6-11 \AA$ with the step of $1 \AA$. We did not use the third direction, which was implemented in [10]. Finally, we obtained 54 profiles, instead of 2,511 in full method [10]; no fuzzy logic was used to reduce the number of profiles as in [10]. Then, the energies of 54 profiles were 
calculated. For each segment from the dataset the energy was obtained with Rosetta Design program $[45,46]$, which added the side chains to the backbones, applied a random component of the simulated annealing to relax the structure, and calculated the energy of infinite periodic system (for more details of the energy calculation procedure see [45]). As an optimal configuration, for each hexapeptide a structure with minimal energy was selected from the set of profiles. Similarly as in [10], the threshold of $-23 \mathrm{kcal} /$ mol was assumed to classify amylogenicity of a segment. Positive instances contained at least one chain whose energy was not greater than the threshold.

The full original 3D profile method, evaluating a single 6-residue segment, required $18-20 \mathrm{CPU}$-hours $(2.5 \mathrm{GHz}$ AMD Opteron, Phenom or Intel Xeon CPU), while 2-2.5 CPU hours were needed with the triplet method and fuzzy logic selecting 80-100 templates [11], supplement. Our method applies the original method, only reducing the number of templates 46 times (from 2500 to 54). The energy calculation would take 0.5 CPU-hour for each hexapeptide, with the same computer.

\section{Machine learning}

The classifiers were trained on all 4481 hexapeptides from our dataset obtained by simplified 3D profile method (Additional file 1). Prediction methods were provided by WEKA 3.6.6 (Waikato Environment for Knowledge Analysis) [27],which includes a hundred of different classifiers. Pre-selection, to find the most effective methods, was carried out with default WEKA sets of parameters and 10-fold cross-validation on the training set. Next, we chose 10 most promising methods from WEKA suite. Finally, we used the following methods with the optimized parameters:

- ADTree (numOfBoostingInterations $=250$ or 50 , randomSeed $=1)$,

- BFTree $(\operatorname{minNumObj}=4$, numFoldsPruning $=4$, seed $=2$, pruningStrategy $=$ un-pruned),

- FT (minNumInstances $=26$, numBoostingIterations $=86$ ),

- RF (maxDepth=unlimited, numFeatures $=\log 2(7)+1$, numTrees $=200$, seed $=1$ ),

- JRip (folds=4, $\operatorname{minNo}=1$, optimizations $=7$, seed $=4$ ),

- MLP (hiddenLayers=1 with 60 nodes, learningRate $=0.1$, Momentum $=0.2$, seed $=0$, training Time $=500$ ),

- PART (confidenceFactor $=0.3$, mniNumObj $=1$, numFold $=3$, seed $=1$ ),

- Ridor (folds $=3$, $\operatorname{minNo}=6$, seed $=1$, shuffle $=7$ ),

- SVM (c=2.0, kernel=linear)

- Naive Bayes (no parameters).

Parameters not specified have their values set to default.
Out of the above mentioned methods, ADTree algorithm has several advantages over other machine learning methods, such as MLP or SVM, including easy interpretation of the results. An alternating decision tree is in fact a graphical representation of a collection of user interpretable rules. Each tree consists of decision and prediction nodes. The prediction node contains a single number, whereas decision node defines a predicate conditions. To classify an instance, the ADTree follows all paths for which all decision nodes are true. The final classification score is gained by summing all the prediction nodes through which the instance it passes.

\section{Prediction accuracy assessment}

The binary test set included 346 positive and 1240 negative hexapeptides from ZipperDB, as of May 2010 (Additional file 1). The classification results were evaluated based on typical measures: Sensitivity (called True Positive Rate - TPR), Specificity (True Negative Rate TNR), Accuracy (Acc). These criteria widely used to evaluate the performance of prediction models, and defined as below:

$$
\begin{gathered}
T P R=\mathrm{TP} /(\mathrm{TP}+\mathrm{FN}) \\
\mathrm{TNR}=\mathrm{TN} /(\mathrm{TN}+\mathrm{FP}) \\
\text { Accuracy }=(\mathrm{TP}+\mathrm{TN}) /(\mathrm{TP}+\mathrm{TN}+\mathrm{FP}+\mathrm{FN})
\end{gathered}
$$

where TP, FP, FN and TN represent the numbers of true positives, false positives, false negatives and true negatives respectively. The overall quality of a classifier can be evaluated with area under ROC curve (AUC) [47]. The value of the AUC score ranges from zero to one, with a score of 0.5 corresponding to random guess and a score of 1.0 indicating perfect separation. This estimator evaluates the method in separating amyloids from non-amyloids. In particular, The AUC is well received in the imbalanced dataset community and it is becoming the standard evaluation method.

\section{Statistical validation}

In order to obtain a representative estimate of WEKA methods performance, which is independent of class distribution and the specifics of the training data set, we performed an experiment with 10 train and test runs. The data used for training and testing - a dataset of 4481 hexapeptides - was randomly divided into $66 \%$ and $34 \%$, respectively. The results were analysed statistically using a corrected paired samples t-test [46] where we computed p-values at the $5 \%$ significance level, comparing every method with every other method for their AUC. This is a parametric procedure used to determine whether there is a significant difference between the average values of the same performance measure for two different methods. The test assumes that the paired differences are 
independent and identically normally distributed. Although the measurements themselves may not be normally distributed, the pair wise differences often are.

\section{Validation with other classification methods}

Two different state of the art methods were used to test the inter-compatibility of the energy based method: FoldAmyloid [18,48] and Waltz [23,49] (as of March 2012). We performed the analysis on the whole set of 4481 hexapeptides, using our scripts in Python programming language and spynner - open source web browsing module for communication with both services. All standard FoldAmyloid methods were applied: contacts, bonebone donors, bone-bone acceptors, hybrid (contacts + donors), and triple hybrid (contacts + donors + acceptors). Waltz was run with its standard optimizations for overall performance and sensitivity.

\section{Additional files}

Additional file 1: Dataset of hexapeptides with calculated energies and amylogenic classification.

Additional file 2: Position specific aminoacid frequencies of the training and test datasets.

Additional file 3: Detailed results of the best machine learning methods trained on the full and reduced training sets.

Additional file 4: Rules of ADTree $\mathbf{2 5 0}$ methods for full and reduced training sets. Columns contain the rules corresponding to each position in amyloidogenic hexapeptides in decreasing order of their importance. Yellow cells denote positive rules, purple - negative rules.

Additional file 5: Amylogenic classification of our dataset obtained with reduced 3D profile (additional file 1: trainset(+) and trainset(-)) with different methods: 3D profile, FoldAmyloid and Waltz. The file includes the spreadsheets labeled according to the name of the external method.

\section{Competing interests}

The authors declare that they have no competing interests.

\section{Authors' contributions}

JS proposed the simplified 3D profile method, generated the dataset and did preliminary tests on the machine learning methods. MK validated the dataset with ZipperDB, ran and analyzed machine learning, FoldAmyloid and Waltz methods, and drafted the manuscript. OU ran and analyzed machine learning methods, performed the statistical analysis, and participated in writing the manuscript. MK and OU designed and supervised the study. All authors read and approved the final manuscript.

\section{Acknowledgements}

This work was in part supported by the grant N N519 643540 from National Science Center of Poland. Wroclaw Centre for Networking and Supercomputing at Wroclaw University of Technology is greatly acknowledged.

Received: 22 May 2012 Accepted: 19 December 2012

Published: 17 January 2013

\section{References}

1. Jaroniec CP, MacPhee CE, Bajaj VS, McMahon MT, Dobson CM, Griffin RG: High-resolution molecular structure of a peptide inan amyloid fibril determined by magic angle spinning NMR spectroscopy. Proc Natl Acad Sci USA 2004, 101:711-716.
2. Makin OS, Atkins E, Sikorski P, Johansson J, Serpell LC: Molecular basis for amyloid fibril formation and stability. Proc Natl Acad Sci USA 2005, 102:315-320.

3. Nelson R, Sawaya MR, Balbirnie M, Madsen AO, Riekel C, Grothe R, Eisenberg D: Structure of the cross- beta spine of amyloid-like fibrils. Nature 2005, 435:773-778.

4. Sawaya MR, Sambashivan S, Nelson R, Ivanova MI, Sievers SA, Apostol MI, Thompson MJ, Balbirnie M, Wiltzius JJW, McFarlane HT, Madsen AØ, Riekel C, Eisenberg D: Atomic structures of amyloid cross $\beta$-spines reveal varied steric zippers. Nature 2007, 447:453-457.

5. Uversky VN, Fink AL: Conformational constraints for amyloid fibrillation: the importance of being unfolded. Biochim Biophys Acta 2004, 1698:131-153.

6. Fowler DM, Koulov AV, Balch WE, Kelly JW: Functional amyloid- from bacteria to humans. Trends Biochem Sci 2007, 32:217-224.

7. Rousseau F, Schymkowitz J, Serrano L: Protein aggregation and amyloidosis: confusion of the kinds? Curr Opin Struct Biol 2006, 16:118-126.

8. Lopez de la Paz M, Serrano L: Sequence determinants of amyloid fibril formation. Proc Natl Acad Sci USA 2004, 101:87-92.

9. Fernandez-Escamilla AM, Rousseau F, Schymkowitz J, Serrano L: Prediction of sequence-dependent and mutational effects on the aggregation of peptides and proteins. Nat Biotechnol 2004, 22:1302-1306.

10. Thompson MJ, Sievers SA, Karanicolas J, Ivanova MI, Baker D, Eisenberg D: The 3D profile method for identifying fibril-forming segments of proteins. Proc Natl Acad Sci U S A 2006, 103:4074-4078.

11. Goldschmidt L, Tenga PK, Riek R, Eisenberg D: Identifying the amylome, proteins capable of forming amyloid-like fibrils. PNAS 2010, 107:3487-3492.

12. Trovato A, Seno F, Tosatto SC: The PASTA server for protein aggregation prediction. Protein Eng Des Sel 2007, 20:521-523.

13. Conchillo-Solé O, de Groot NS, Avilés FX, Vendrell J, Daura X, Ventura S: AGGRESCAN: a server for the prediction and evaluation of "hot spots" of aggregation in polypeptides. BMC Bioinformatics 2007, 8:65.

14. Zhang Z, Chen H, Lai L: Identification of amyloid fibril-forming segments based on structure and residue-based statistical potential. Bioinformatics 2007, 23:2218-2225.

15. Tartaglia GG, Vendruscolo M: The Zyggregator method for predicting protein aggregation propensities. Chem Soc Rev 2008, 37:1395-1401.

16. Tartaglia GG, Vendruscolo M: Proteome-level interplay between folding and aggregation propensities of proteins. J Mol Biol 2010, 402:919-928.

17. Kim C, Choi J, Lee SJ, Welsh WJ, Yoon S: NetCSSP: web application for predicting chameleon sequences and amyloid fibril formation. Nucleic Acids Res 2009, 37:W469-W473.

18. Garbuzynskiy SO, Lobanov MY, Galzitskaya OV: FoldAmyloid: a method of prediction of amyloidogenic regions from protein sequence. Bioinformatics 2010, 26:326-332.

19. O'Donnell CW, Waldispühl J, Lis M, Halfmann R, Devadas S, Lindquist S, Berger B: A method for probing the mutational landscape of amyloid structure. Bioinformatics 2011, 27:i34-i42.

20. Bryan AW Jr, O'Donnell CW, Menke M, Cowen LJ, Lindquist S, Berger B: STITCHER: Dynamic assembly of likely amyloid and prion $\beta$-structures from secondary structure predictions. Proteins 2011, 80:410-420.

21. Bryan AW Jr, Menke M, Cowen LJ, Lindquist SL, Berger B: BETASCAN: probable beta-amyloids identified by pairwise probabilistic analysis. PLOS Comput Biol 2009, 5:e1000333.

22. Frousios KK, Iconomidou VA, Karletidi CM, Hamodrakas SJ: Amyloidogenic determinants are usually not buried. BMC Struct Biol 2009, 9:44.

23. Maurer-Stroh S, Debulpaep M, Kuemmerer N, Lopez de la Paz M, Martins IC, Reumers J, Morris KL, Copland A, Serpell L, Serrano L, Schymkowitz JW, Rousseau F: Exploring the sequence determinants of amyloid structure using position-specific scoring matrices. Nat Methods 2010, 7:237-242.

24. David MP, Concepcion GP, Padlan EA: Using simple artificial intelligence methods for predicting amyloidogenesis in antibodies. BMC Bioinformatic 2010, 11:79.

25. http://services.mbi.ucla.edu/zipperdb/.

26. Witten $\mathrm{H}$ : Frank EL Data Mining: Practical machine learning tools and techniques, 2nd Edition ed. San Francisco: Morgan Kaufmann; 2005.

27. Hall M, Frank E, Holmes G, Pfahringer B, Reutemann P, Witten $\mathbb{H}_{\text {: }}$ The WEKA Data Mining Software: An Update; SIGKDD Explorations. 2009, 11(1):10-18 
28. Freund $Y$, Mason L: The Alternating Decision Tree Learning Algorithm. Proceedings of the 16th International Conference on Machine Learning, Bled, Slovenia; 1999:124-133.

29. Itti L, Koch C, Niebur E: A model of saliency-based visual attention for rapid scene analysis. IEEE Trans Pattern Anal Mach Intell 1998, 20(11):1254-1259.

30. Gama J: Functional trees. Machine Learning 2004, 55(3):219.

31. Cohen WW: Fast Effective Rule Induction. Proceedings of the 12th International Conference on Machine Learning: Morgan Kaufmann Publishers; 1995:115-123.

32. Frank E, Witten $\mathbb{I H}$ : Generating Accurate Rule Sets Without Global Optimization. In Proceedings of the 15th International Conference on Machine Learning. Edited by Shavlik J: Morgan Kaufmann Publishers; 1998:144-151.

33. Gaines BF, Compton P: Induction of ripple-down rules applied to modeling large databases. Journal of Intelligent Information Systems 1995, 5(3):211-228.

34. Platt JC: Fast Training of Support Vector Machines Using Sequential Minimal Optimization. In Advances in Kernel Methods - Support Vector Learning. Edited by Schölkopf B, Burges C, Smola A. Cambridge, MA: MIT Press; 1999:185-208.

35. Rumelhart D, Hinton G, Williams R: Learning Internal Representations by Error Propagation. Parallel Distributed Processing. Cambridge MA: MIT Press; 1986:318-362.

36. John GH, Langley P: Estimating Continuous Distributions in Bayesian Classifiers. Proceedings of the 11th Conference on Uncertainty in Artificial Intelligence: Morgan Kaufmann Publishers; 1995:338-345.

37. Breiman L: Random Forests. Machine Learning 2001, 45(1):5-32.

38. http://www.uniprot.org/.

39. Pawlicki S, Le Béchec A, Delamarche C: AMYPdb: a database dedicated to amyloid precursor proteins. BMC Bioinformatics 2008, 9:273.

40. http://amypdb.univ-rennesi.fr.

41. Li W, Godzik A: Cd-hit: a fast program for clustering and comparing large sets of protein or nucleotide sequences. Bioinformatics 2006, 22:1658-1659.

42. http://weizhong-lab.ucsd.edu/cd-hit/.

43. http://web.expasy.org/docs/relnotes/relstat.html.

44. Crooks GE, Hon G, Chandonia JM, Brenner SE: WebLogo: a sequence logo generator. Genome Res 2004, 14:1188-1190.

45. Kuhlman B, Baker D: Native protein sequences are close to optimal for their structures. Proc Natl Acad Sci U S A 2000, 97:10383-10388.

46. http://www.rosettacommons.org/.

47. Hanley J, McNeil B, et al: A method of comparing the areas under receiver operating characteristic curves derived from the same cases. Radiology 1983, 148:839-843

48. http://bioinfo.protres.ru/fold-amyloid/oga.cgi.

49. http://waltz.switchlab.org/.

doi:10.1186/1471-2105-14-2

Cite this article as: Stanislawski et al:: Machine learning methods can replace 3D profile method in classification of amyloidogenic hexapeptides. BMC Bioinformatics 2013 14:21.

\section{Submit your next manuscript to BioMed Central and take full advantage of:}

- Convenient online submission

- Thorough peer review

- No space constraints or color figure charges

- Immediate publication on acceptance

- Inclusion in PubMed, CAS, Scopus and Google Scholar

- Research which is freely available for redistribution

Submit your manuscript at www.biomedcentral.com/submit 